\title{
Invasive Pulmonary Aspergillosis after COVID-19 Pneumonia
}

\author{
Filipa David $^{1}$, Joana Rodrigues Morais ${ }^{1}$, Francisca Beires ${ }^{1}$, Helena Greenfield ${ }^{1}$, Gonçalo L Fernandes ${ }^{2}$ \\ 1 Internal Medicine Department, Hospital Pedro Hispano, Unidade Local de Saúde de Matosinhos, Portugal \\ ${ }^{2}$ Intensive Care Unit, Hospital Pedro Hispano, Unidade Local de Saúde de Matosinhos, Portugal
}

How to cite this article: David F, Rodrigues Morais J, Beires F, Greenfield H, Fernandes GL. Invasive pulmonary aspergillosis after COVID-19 pneumonia. EJCRIM 2022;9:doi:10.12890/2022_003209.

Conflicts of Interests: The Authors declare that there are no competing interests.

This article is licensed under a Commons Attribution Non-Commercial 4.0 License

\section{ABSTRACT}

COVID-19 emerged in China in late 2019 and quickly spread worldwide. The severe immunomodulation and depletion of lymphocytes caused by the virus and its therapy led to an increase in the incidence of superinfections. COVID-19-associated pulmonary aspergillosis (CAPA) is a new entity with increasing incidence and high associated mortality.

We present the case of a 68-year-old patient admitted to our ward after recovering from severe COVID-19 pneumonia. Due to worsening of her clinical condition, chest computed tomography was performed and a lung abscess was documented with the identification of Aspergillus niger. Despite therapy with voriconazole, the patient's condition deteriorated, culminating in her death.

\section{KEYWORDS}

COVID-19, pulmonary aspergillosis, Aspergillus niger

\section{LEARNING POINTS}

- COVID-19-associated pulmonary aspergillosis (CAPA) is a new entity with an increasing incidence.

- It is a serious and life-threatening complication in patients with severe COVID-19 even in the absence of the classic risk factors for invasive pulmonary aspergillosis.

- Clinical suspicion is crucial since a timely diagnosis and treatment have a major impact on prognosis.

\section{CASE DESCRIPTION}

A 68-year-old woman with vascular risk factors (arterial hypertension, hypertriglyceridemia and grade I obesity) and a week-old diagnosis of SARS-CoV-2 infection, presented to the emergency department with fever, non-productive cough and fatigue over the previous 4 days. She was apyretic, haemodynamically stable and tachypnoeic, with a diffusely diminished vesicular murmur and crackles in both lungs. Severe hypoxemic respiratory failure was documented $\left(\mathrm{pO}_{2} / \mathrm{FiO}_{2}\right.$ ratio <100). She presented a CRP of $189.90 \mathrm{mg} / \mathrm{l}$, ferritin of $955.11 \mathrm{ng} /$ $\mathrm{ml}$, LDH of $426 \mathrm{U} / \mathrm{l}$ and D-dimer level of $815.5 \mathrm{ng} / \mathrm{ml}$ as parameters predictive of a poor outcome in COVID-19. A computed tomography angiography (angio-CT) scan of the chest revealed ground-glass areas covering more than $75 \%$ of the lung parenchyma, compatible with COVID-19 pneumonia (Fig. 1), without areas of cavitation or signs of pulmonary thromboembolism. She was admitted to the intensive care unit and underwent therapy with dexamethasone $16 \mathrm{mg}$ i.v. once daily, and organ support measures, namely mechanical ventilation, with good response and no need for prone positioning.

On the 8th day of hospitalization, the patient presented with a recurrence of fever, purulent bronchial secretions, and a worsening of her inflammatory parameters (Table 1). 
European Journal

of Case Reports in

Internal Medicine

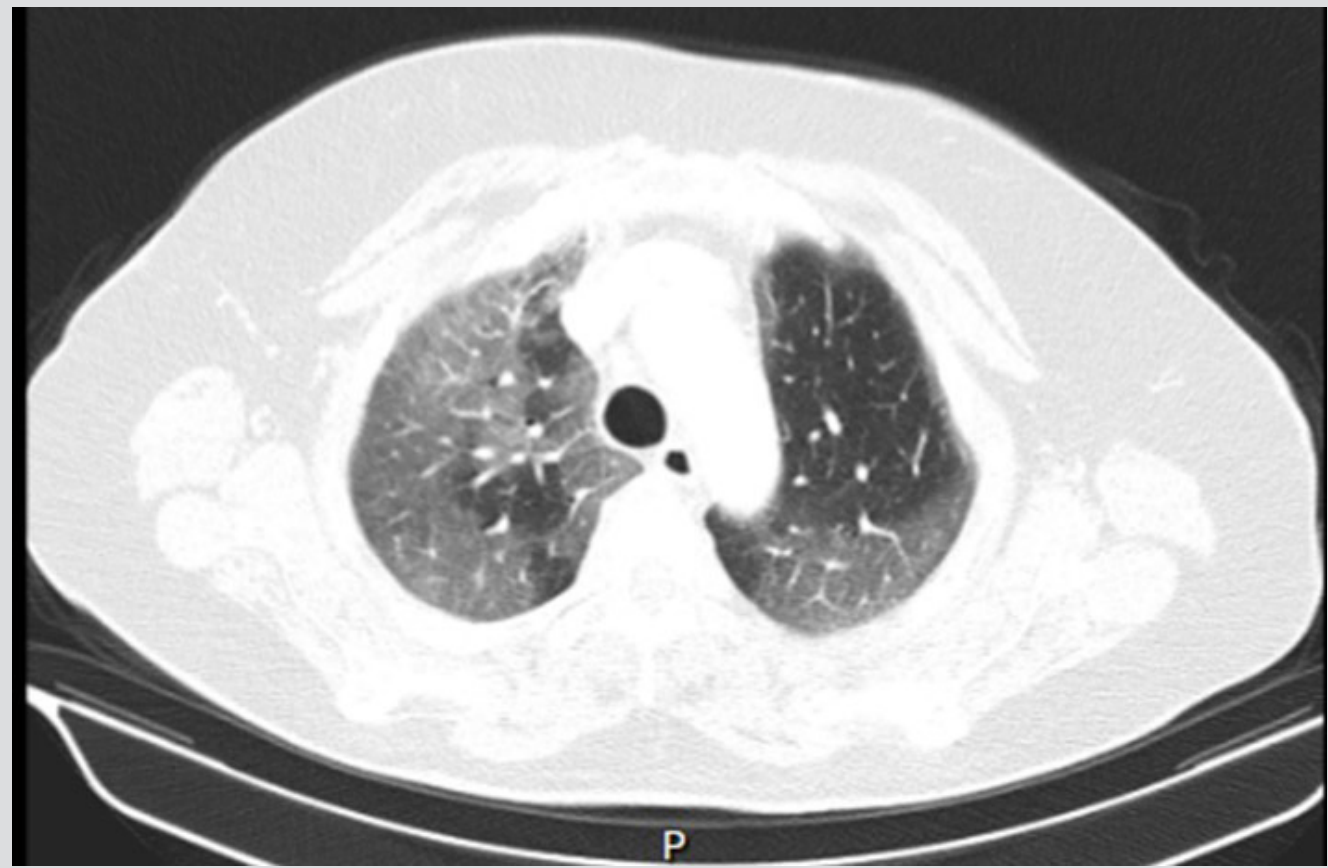

Figure 1. Chest angio-CT scan showing ground-glass areas, without areas of cavitation

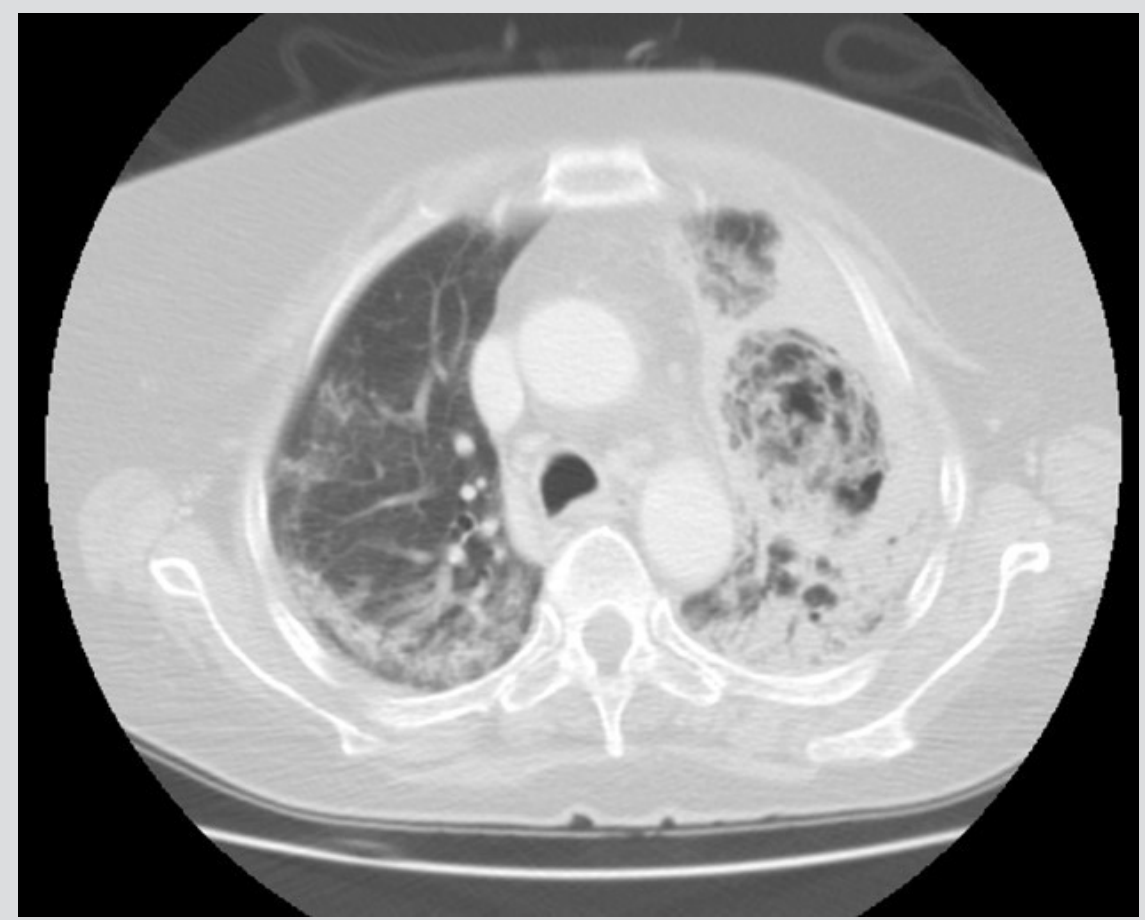

Figure 2. Chest CT angiography showing a necrotic lesion compatible with an abscess, measuring $61 \times 50 \mathrm{~mm}$, with heterogeneous content and thick walls, in the left upper lobe

Enterobacter cloacae spp. was isolated in tracheal aspirate and acute tracheobronchitis was diagnosed, and the patient was treated with a 5 -day course of piperacillin /tazobactam $4.5 \mathrm{mg}$ every 8 hours. She evolved positively, allowing gradual ventilatory weaning, with extubation after 7 days, and step-down of care with subsequent transfer to the general ward.

On the 14th day of hospitalization, while still being weaned off corticotherapy (prednisolone 20 mg once a day, by mouth), a new upsurge of inflammatory parameters was noted, as well as non-specific clinical presentation of cough with mucous expectoration and fatigue. Despite 


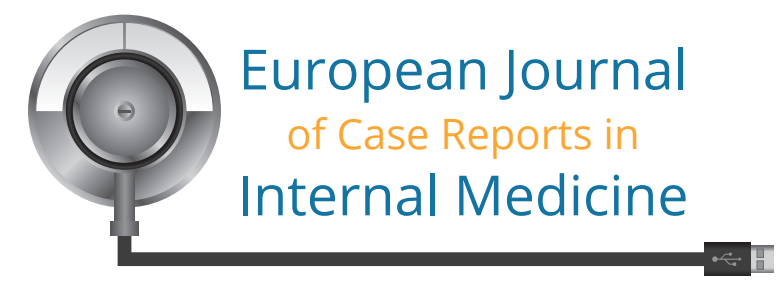

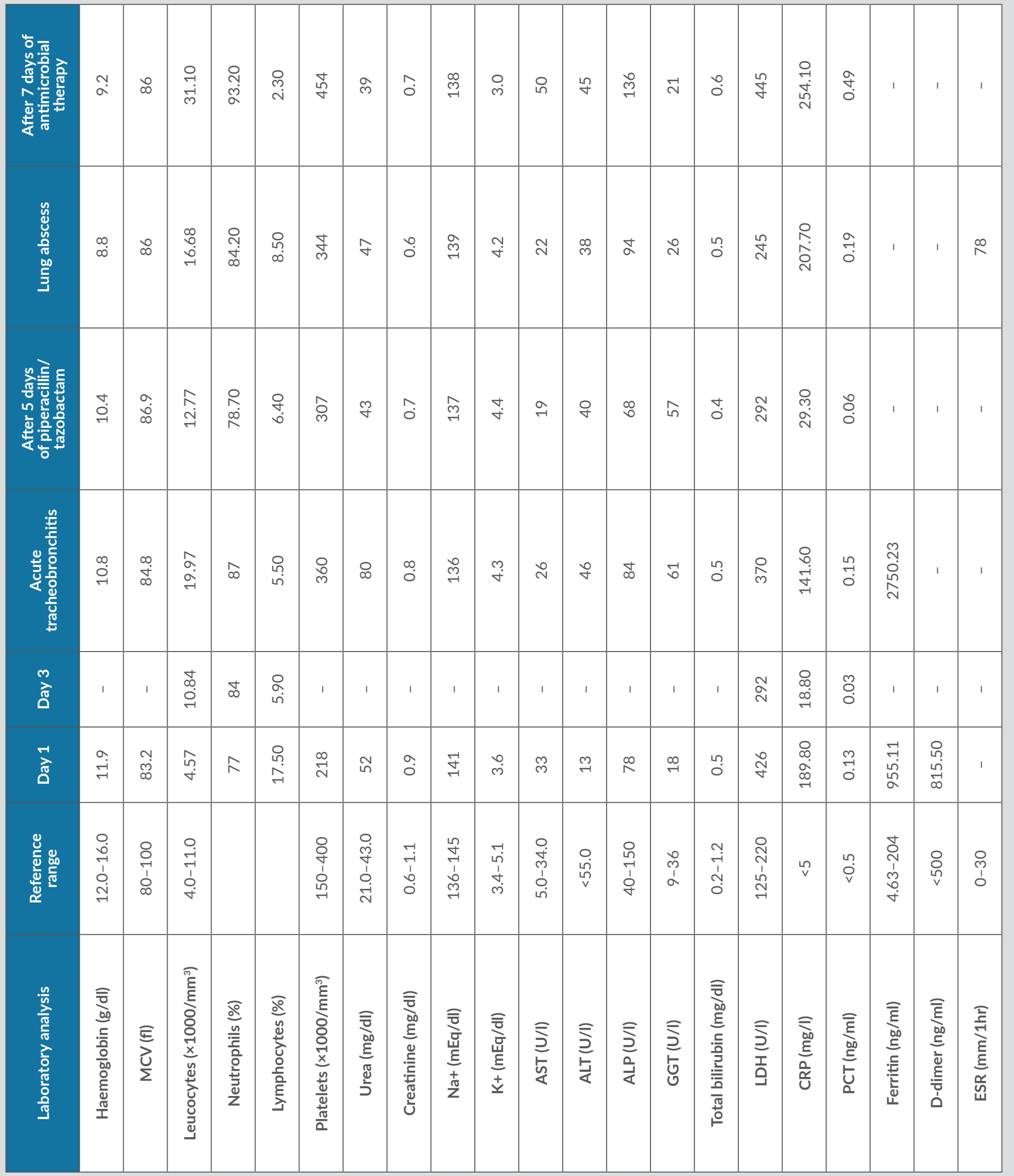


clinical stability, the persistent inflammatory syndrome led to a new chest CT scan (Fig. 2) that documented an abscess in the upper lobe of the left lung with areas of peripheral consolidation.

Bronchoscopy and a pulmonary biopsy were performed with identification of Aspergillus niger, permitting the diagnosis of invasive pulmonary aspergillosis. In spite of broad-spectrum antibiotics (meropenem $1 \mathrm{~g}$ every 8 hours and linezolid 600 mg every 12 hours) and voriconazole $300 \mathrm{mg}$ every 12 hours, the patient deteriorated, with multiple organ failure culminating in death.

\section{DISCUSSION}

With the arrival of the COVID-19 pandemic, medicine evolved rapidly in an attempt to understand the pathophysiology of the SARS-CoV-2 infection, and thus identify possible therapeutic options. After 2 years of research, it is known that COVID-19 is an infection marked by a large release of cytokines such as TNF-alpha and IL-6, leading to a pro-inflammatory state that sometimes mimics the behaviour of sepsis or acute respiratory distress syndrome ${ }^{[1,2]}$. Due to this inflammatory condition, corticosteroids are the most widely used treatment and help control the hyperinflammatory state and manifestations caused by this infection.

COVID-19 has been associated with an immunosuppressive state, which is exacerbated by corticosteroid treatment, resulting in a greater propensity to develop co-infections (bacterial, viral or fungal) ${ }^{[1,3]}$.

CAPA is a new entity, with an incidence ranging from $19 \%$ to $33 \%{ }^{[3]}$ in critically ill patients infected with COVID-19, most of whom have passed through the ICU, requiring a period of mechanical ventilation, and under corticotherapy. In addition to mechanical ventilation and corticosteroid treatment, the presence of comorbidities also predisposes to the development of this fungal infection, but not the classic factors for invasive pulmonary aspergillosis, such as haematological malignancy or neutropenia ${ }^{[1,4]}$.

Unlike other co-infections, such as influenza, Aspergillus infection is thought to manifest late, from 1 week to 15 days after ICU admission ${ }^{[1,5]}$. The timely diagnosis of this entity is a challenge since the clinical features are non-specific ${ }^{[1,4]}$. This difficulty leads to a delay in the investigation and identification of the pathogenic agent, and consequently in the start of treatment, with very high mortality rates of around 40\%. Studies have shown that mortality from CAPA is higher than mortality from COVID-19 alone ${ }^{[2]}$.

This case highlights the need for clinical suspicion so as to make a timely diagnosis, leading to early treatment. Our patient was treated with voriconazole, which is the first-line treatment for $\mathrm{CAPA}^{[3,4]}$, however, the delay in diagnosis and thus treatment initiation led to disease progression and an unfavourable outcome.

\section{REFERENCES}

1. Machado M, Valerio M, Álvarez-Uría A, Olmedo M, Veintimilla C, Padilla B, et al. Invasive pulmonary aspergillosis in the COVID-19 era: an expected new entity. Mycoses 2021 Feb;64(2):132-143.

2. Dewi IM, Janssen NA, Rosati D, Bruno M, Netea MG, Brüggemann RJ, et al. Invasive pulmonary aspergillosis associated with viral pneumonitis. Curr Opin Microbiol 2021 Aug;62:21-27.

3. Lai C-C, Yu W-L. COVID-19 associated with pulmonary aspergillosis: a literature review. J Microbiol Immunol Infect 2021 Feb;54(1):46-53.

4. El-Baba F, Gao Y, Soubani AO. Pulmonary aspergillosis: what the generalist needs to know. Am J Med 2020 Jun;133(6):668-674.

5. Wauters J, Lamoth F, Rijnders BJ, Calandra T. Invasive pulmonary aspergillosis goes viral again? Am J Respir Crit Care Med 2021 Feb 1;203(3):275-277. 